\title{
Dissipative effects in fission investigated with proton-on-lead reactions
}

\author{
J. L. Rodríguez-SÁnchez ${ }^{1 *}$, J. Benlliure ${ }^{1}$, J. TAÏEB $^{2}$, \\ H. Álvarez-Pol ${ }^{1}$, L. Audouin ${ }^{3}$, Y. Ayyad ${ }^{1}$, G. Bélier ${ }^{2}$, \\ G. Boutoux ${ }^{2}$, E. Casarejos ${ }^{4}$, A. Chatillon ${ }^{2}$, D. Cortina-Gil ${ }^{1}$, \\ T. Gorbinet ${ }^{2}$, A. Heinz ${ }^{5}$, A. Kelić-Heil ${ }^{6}$, B. Laurent ${ }^{2}$, \\ J.-F. Martin ${ }^{2}$, C. Paradela ${ }^{1}$, E. Pellereau ${ }^{2}$, B. Pietras ${ }^{1}$, \\ D. Ramos ${ }^{1}$, C. Rodríguez-Tajes ${ }^{7}$, D. M. Rossi ${ }^{6}$, H. Simon $^{6}$, \\ J. VARGAS ${ }^{1}$ and B. $\operatorname{Voss}^{6}$ \\ ${ }^{1}$ Universidad de Santiago de Compostela, E-15782 Santiago de \\ Compostela, Spain \\ 2 CEA, DAM, DIF, F-91297 Arpajon, France \\ ${ }^{3}$ Institut de Physique Nucléaire d'Orsay, F-91406 Orsay, France \\ ${ }^{4}$ Universidad de Vigo, E-36200 Vigo, Spain \\ ${ }^{5}$ Chalmers University of Technology, SE-412 96 Gothenburg, Sweden \\ ${ }^{6}$ GSI Helmholtzzentrum für Schwerionenforschung GmbH, D-64291 \\ Darmstadt, Germany \\ 7 Grand Accélérateur National d'Ions Lourds, F-14076 Caen Cedex 5, \\ France
}

\begin{abstract}
The complete kinematic measurement of the two fission fragments permitted us to investigate dissipative effects at large deformations, between the saddle-point and the corresponding scission configurations. Up to now, this kind of study has only been performed with fusionfission reactions using a limited number of observables, such as the mass distribution of the fission fragments or the neutron multiplicities. However, the large angular momenta gained by the compound nucleus
\end{abstract}

\footnotetext{
*joseluis.rodriguez.sanchez.phy@gmail.com
} 
could affect the conclusions drawn from such experiments. In this work, the use of spallation reactions, where the fissioning systems are produced with low angular momentum, small deformations and high excitation energies, favors the study of dissipation, and allowed us to define new observables, such as postscission neutron multiplicities and the neutron excess of the final fission fragments as a function of the atomic number of the fissioning system. These new observables are used to investigate the dissipation at large deformations.

\section{Introduction}

It is known that the gross features of a fissioning system can be described in terms of a small number of parameters, the so-called collective degrees of freedom, where the dissipation represents the average effect of the interactions between the collective and intrinsic degrees of freedom. In this picture, the interaction between these degrees of freedom results in a the fluctuating force acting on the collective dynamics, which in effect causes the diffusion of the dynamical coordinates (elongation, mass asymmetry, etc.) [1].

However, other questions such as the onset of dissipation and the temperature or deformation dependences of the dissipation parameter are still under debate [2]. A systematic study was carried out by Thoennessen et al. [3] to find the threshold excitation energy at which the statistical model starts losing its validity. Their work opened up the problem of understanding the properties of nuclear dissipation and its dependence on the excitation energy. It was found that the ratio of the threshold temperature $\left(T_{\text {threshold }}\right)$ and the temperature-dependent fission barrier $\left(B_{f}\right)$ is independent of the mass of the fissioning nucleus and equal to 0.26 . The authors suggested that this could reflect an onset of dissipation with increasing temperature. According to this conclusion, Hofman and collaborators [4] found an analytical expression for the temperature dependence of dissipation from the study of the $\gamma$-ray multiplicities associated with several fusion-fission and quasifission reactions. However, the authors of Ref. [5] showed that this systematic behaviour could be explained without assuming an onset of dissipation effects or a temperature dependence of it. This work also pointed out that the reason why the statistical model fails to reproduce the experimental data for $T_{\text {threshold }} / B_{f}>0.26$ is that at that point the dynamical delay time starts to be larger than the decay time predicted by the statistical model. Similar conclusions were also found by Lestone et al. [6,7], who were able to describe evaporation and fission cross sections as well as light-charged particles multiplicities of fusion reactions considering a temperature independence of the 
dissipation parameter.

Recently, Jurado et al. [8], Schmitt et al. [9], and Ayyad et al. [10,11] investigated dissipative effects in fission by using spallation and fragmentation reactions. In these works, both fission fragments were measured with a novel experimental approach [12], similar to the one used in this work [13], which allowed to investigate observables sensitive to dissipation at small deformations, such as the total and partial fission cross sections, and the width of the charge distribution of the fission fragments. The authors demonstrated that dissipative and transient time effects are needed to describe the data, leading to a constant value of the reduced dissipation parameter at small deformations of $\beta=4.5 \times 10^{21} \mathrm{~s}^{-1}$. Moreover, the overall good description of the data also validated the conclusions on the temperature independence of the dissipation parameter. These conclusions, obtained with respect to the magnitude and temperature independence of the reduced dissipation parameter at small deformations, were also confirmed by us investigating the same observables $[14,15]$.

On the other hand, recent works concerning the study of $\gamma$-ray multiplicities in fusion-fission reactions were interpreted assuming a deformation dependence of dissipation instead of a temperature dependence. Using this hypothesis, Shaw and collaborators [16] were able to reproduce the $\gamma$-ray multiplicity emitted in the fission process of ${ }^{240} \mathrm{Cf}$ at different energies by using a value of $\gamma_{\text {ground-saddle }}=2$ inside the saddle point, and of $\gamma_{\text {saddle-scission }}=5-10$ beyond the saddle point ${ }^{1}$. Diószegi and coworkers [17] were able to investigate dissipation at small and large deformations by separating the presaddle and the saddle-to-scission $\gamma$-ray components. They used evaporation-residue cross sections of the reaction ${ }^{9} \mathrm{~F}$ $+{ }^{181} \mathrm{Ta}$ at 161 and $181 \mathrm{MeV}$ to determine the dissipative parameter up to the saddle point, $\gamma_{\text {ground-saddle }}=3$. Fixing this value of the dissipation coefficient at the saddle-point deformation, they varied the dissipation coefficient from saddle to scission to reproduce the $\gamma$-ray multiplicities obtaining $\gamma_{\text {saddle-scission }}=10$. Such a deformation dependence with very low values of dissipation for compact shapes up to the saddle point seems to be compatible with the results obtained by Fröbrich and collaborators [18]. These authors analyzed the prescission neutron multiplicities and fission probabilities with one-dimensional Langevin calculations, coupled to a statistical evaporation code. They found that the experimental observables could only

\footnotetext{
${ }^{1}$ Note that $\gamma_{i}$ (where the subscript $i$ denotes ground-saddle or saddle-scission) is related with the reduced dissipation parameter according to $\gamma_{i}=\beta_{i} / 2 \omega$, being $\omega$ the frequency of the inverted oscillator potential at the saddle point.
} 
be described with a deformation-dependent dissipative parameter by considering a constant value $\beta_{\text {ground-saddle }}=2 \times 10^{21} \mathrm{~s}^{-1}$ at small deformations, and a linear increase with deformation when the fissioning system descends from the saddle to the scission point.

In order to contribute to this discussion, we take advantage of the complete characterization of both fission fragments produced in proton-induced fission reactions on ${ }^{208} \mathrm{~Pb}$ at $500 \mathrm{~A} \mathrm{MeV}$. The measurement was performed by using the SOFIA setup $[19,20]$ mounted at the GSI facilities. The inverse kinematics used in this experiment gave us, for the first time, access to the determination of the velocities, atomic and mass numbers of both fission fragments [13] simultaneously. With these measurements, we can define observables sensitive to dissipation, such as the neutron excess of the fission fragments, constraining the value of the dissipation parameter at large deformations by using model calculations.

\section{Model calculations}

The calculations were done by using the code INCL4.6 [21] to describe the first stage of the reaction induced by energetic protons according to the intranuclear cascade approach. The deexcitation of the resulting remnants was described by employing the code ABLA07 [22]. This statistical code describes the emission of $\gamma$-rays, neutrons, light-charged particles and intermediate-mass fragments (IMFs) according to the Weisskopf formalism [23], which provides a good description of the evaporation residues produced in spallation and fragmentation reactions of nuclei from iron to uranium $[21,24-26]$. The fission decay width is described by an analytical approximation of the solution of the Fokker-Planck equation, describing fission as a diffusion process across the fission barrier [27]. Fission barriers are calculated according to the finite-range liquid-drop model of Sierk [28]. Finally, if fission is reached, the mass- and atomic-number distributions of the fission fragments are described by the semiempirical model proposed in Ref. [29].

In order to include the fission dynamics beyond the saddle point, the saddle-to-scission time, needed by the fissioning system to descend from the saddle to the scission point, can be compared with the statistical evaporation time obtained from the neutron decay width. If the saddle-to-scission time of the fissioning system is longer than the statistical evaporation time, the fissioning system could emit neutrons during that phase, increasing the prescission neutron multiplicities. With the aim of introducing these dy- 
namical effects in our calculations, we have used the pioneering theoretical model proposed by Hofmann and Nix [30], who found an analytical solution of the average saddle-to-scission time based on the dynamical picture of Kramers [31]. Following this formalism, the average saddle-to-scission dissipative time $\left(\tau_{s s c}\right)$ can be calculated according to:

$$
\tau_{s s c}=\left(\left[1+\left(\frac{\beta}{2 \omega_{0}}\right)^{2}\right]^{1 / 2}+\frac{\beta}{2 \omega_{0}}\right) \tau_{s s c}^{0}
$$

where $\omega_{0}$ is the frecuency of the inverted oscillator potencial at the saddle point, $\beta$ is the reduced dissipation parameter, and $\tau_{s s c}^{0}$ is the saddle-toscission statistical time.

\section{Results}

The complete kinematic measurement of the two fission fragments was used to reconstruct the postscission neutron multiplicities using an indirect method based on the Wilkins model [32]. The resulting postscission neutron multiplicities are displayed in Fig. 1 as a function of the atomic number of the fissioning system. This observable is compared with different model calculations: assuming no evaporation of particles between the saddle and scission points (dotted lines), calculations based on the saddle-to-scission statistical time $\tau_{s s c}^{0}$ (long-dashed line), and calculations based on Eq. 1 with a dissipation parameter of $\beta=18 \times 10^{21} \mathrm{~s}^{-1}$ (dot-long-dashed line). The latter calculation is displayed to show the sensitivity of this observable to dissipative effects.

From the comparison of the neutron multiplicities with our calculations, one can see that, as expected, the saddle-to-scission dissipative effects do not affect the postscission neutron multiplicities because the multiplicities seem to be very similar for all the fissioning systems. Thus, these results indicate us that the neutron excess of the two final fission fragments could be as sensitive to dissipation at large deformation as the prescission neutron multiplicities. Therefore, we propose using this observable as a function of the atomic number of the fissioning system. As demonstrated in Ref. [13], the neutron excess as a function of the atomic number of the fission fragments provides important information on the partition of the excitation energy between the two fission fragments at scission. However, the neutron excess as a function of the atomic number of the fissioning system should help us to obtain information on the saddle-to-scission dynamics. 


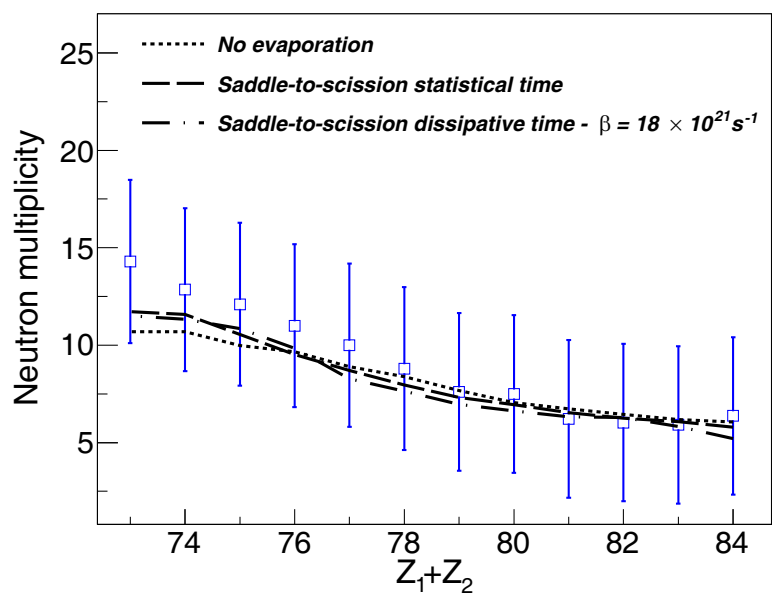

Figure 1: (Color online) The postscission neutron multiplicities are compared with different model calculations (lines).

The average neutron excess of the fission fragments, obtained in Ref. [13], is displayed as a function of the atomic number of the fissioning nuclei in Fig. 2 for the reaction ${ }^{208} \mathrm{~Pb}+p$ at $500 \mathrm{~A} \mathrm{MeV}$. In the figure, we also compare the neutron excess with our previous model calculations. First, the average neutron excess is compared with a calculation assuming the saddleto-scission statistical time $\tau_{s s c}^{0}$ (long-dashed line). As can be observed, this calculation clearly overestimates the neutron excess for the lightest fissioning systems. This overestimation indicates the need for dissipation at high excitation energies where the statistical evaporation time is comparable to the time needed by the fissioning nucleus to descend from the saddle point to scission. Therefore, we also compare the data with dissipative calculations based on Eq. 1, assuming a reduced dissipation parameter $\beta$ of 4.5 $\times 10^{21} \mathrm{~s}^{-1}$ (short-dashed line), $6.5 \times 10^{21} \mathrm{~s}^{-1}$ (solid line), and $18 \times 10^{21}$ (dot-long-dashed line) $\mathrm{s}^{-1}$. As can be seen in the figure, the calculation considering a reduced dissipation parameter of $4.5 \times 10^{21} \mathrm{~s}^{-1}$ (short-dashed line) or $6.5 \times 10^{21} \mathrm{~s}^{-1}$ (solid line) can describe the average neutron excess for all the fissioning systems. These results could indicate that the dissipation parameter does not depend on deformation because, in our previous works, we have determined a dissipation parameter of $(4.5 \pm 1.0) \times 10^{21} \mathrm{~s}^{-1}$ at small deformations $[14,15]$.

Our results seem to contradict most of the works performed with fusionfission reactions [16-18,33-35], where a deformation-dependent dissipation parameter was claimed to describe the prescission particle multiplicities. 
However, the conclusions obtained using fusion-fission reactions could be affected by the initial conditions of the compound nucleus and by the fact that several independent observables are needed for constraining the dissipation parameter accurately.

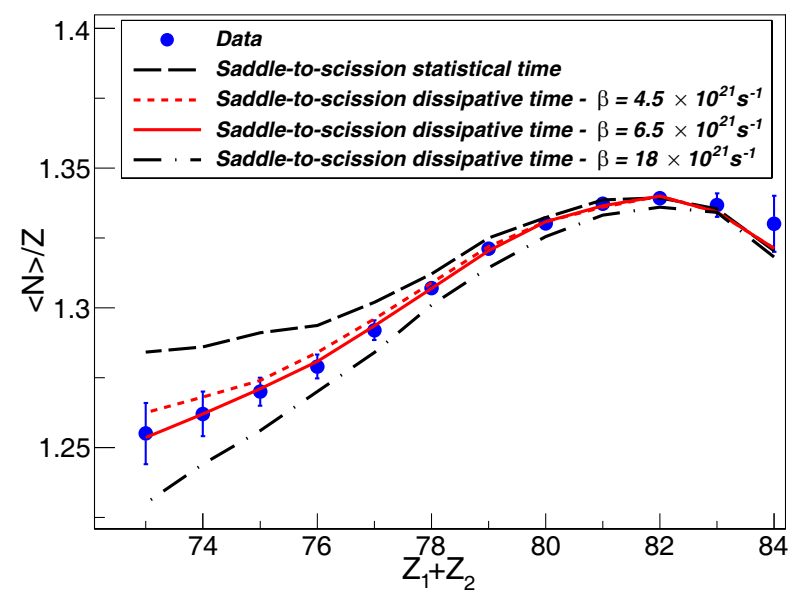

Figure 2: (Color online) The average neutron excess of the final fission fragments produced in the reaction ${ }^{208} \mathrm{~Pb}+p$ at $500 A \mathrm{MeV}$ (solid circles) is compared to different model calculations (lines).

\section{Conclusions}

The complete kinematics measurement of the fission fragments produced in the reaction ${ }^{208} \mathrm{~Pb}(500 \mathrm{~A} \mathrm{MeV})+p$ was used to reconstruct the average postscission neutron multiplicities. This observable, together with the average neutron excess of the final fission fragments, were displayed as a function of the atomic number of the fissioning nucleus to investigate dissipative effects at large deformations. The comparison of different model calculations with these observables shows that the neutron excess is more sensitive to dissipative effects than the average postscission neutron multiplicities. The higher accuracy of the neutron excess allowed us to determine the value of the dissipation parameter at large deformations, obtaining a value of the reduced dissipation parameter of $\beta_{\text {saddle-scission }}=(6.5 \pm 2.5) \times 10^{21} \mathrm{~s}^{-1}$. Taking its uncertainty into account, this value is in agreement with the value of the reduced dissipation parameter obtained at small deformations, 
$\beta_{\text {ground-saddle }}=(4.5 \pm 1.0) \times 10^{21} \mathrm{~s}^{-1}$, and therefore we can conclude that no dependence on deformation is observed.

\section{References}

[1] Y. Abe et al., Phys. Rep. 275, 49 (1996).

[2] D. Jacquet and M. Morjean, Prog. Part. Nucl. Phys. 63, 155 (2009).

[3] M. Thoennessen et al., Phys. Rev. Lett. 71, 4303 (1993).

[4] D. J. Hofman et al., Phys. Rev. C 51, 2597 (1995).

[5] H. van der Ploeg et al., Phys. Rev. Lett. 75, 970 (1995).

[6] J. P. Lestone, Phys. Rev. Lett. 70, 2245 (1993).

[7] J. P. Lestone and S. G. McCalla, Phys. Rev. C 79, 044611 (2009).

[8] B. Jurado et al., Phys. Rev. Lett. 93, 072501 (2004).

[9] C. Schmitt et al., Phys. Rev. C 81, 064602 (2010).

[10] Y. Ayyad et al., Phys. Rev. C 89, 054610 (2014).

[11] Y. Ayyad et al., Phys. Rev. C 91, 034601 (2015).

[12] K.-H. Schmidt et al., Nucl. Phys. A 665, 221 (2000).

[13] J. L. Rodríguez-Sánchez et al., Phys. Rev. C 91, 064616 (2015).

[14] J. L. Rodríguez-Sánchez et al., Phys. Rev. C 90, 064606 (2014).

[15] J. L. Rodríguez-Sánchez et al., accepted in Phys. Rev. C (2015).

[16] N. P. Shaw et al., Phys. Rev. C 61, 044612 (2000).

[17] I. Diószegi et al., Phys. Rev. C 63, 014611 (2001).

[18] P. Fröbrich et al., Nucl. Phys. A 556, 281 (1993).

[19] G. Boutoux et al., Phys. Procedia 47, 166 (2013).

[20] T. Gorbinet et al., Phys. Procedia 64, 101 (2015).

[21] A. Boudard, J. Cugnon, J.-C. David, S. Leray, and D. Mancusi, Phys. Rev. C 87, 014606 (2013). 
[22] A. Kelić, M. V. Ricciardi, and K.-H. Schmidt, Proceedings of Joint ICTP-IAEA Advanced Workshop on Model Codes for Spallation Reactions, ICTP Trieste, Italy, 4-8 February 2008, edited by D. Filges, S. Leray, Y. Yariv, A. Mengoni, A. Stanculescu, and G. Mank (IAEA INDC(NDS)530, Vienna, 2008), pp. 181-221.

[23] V. F. Weisskopf and D. H. Ewing, Phys. Rev. 57, 472 (1940).

[24] M. V. Ricciardi et al., Phys. Rev. C 73, 014607 (2006).

[25] C. Villagrasa-Canton et al., Phys. Rev. C 75, 044603 (2007).

[26] J. Alcántara-Núñez et al., Phys. Rev. C 92, 024607 (2015).

[27] B. Jurado, K.-H. Schmidt, and J. Benlliure, Phys. Lett. B 553, 186 (2003).

[28] A. J. Sierk, Phys. Rev. C 33, 2039 (1986).

[29] J. Benlliure et al., Nucl. Phys. A 628, 458 (1998).

[30] H. Hofmann and J. R. Nix, Phys. Lett. B 122, 117 (1983).

[31] H. A. Kramers, Physica 7, 284 (1940).

[32] B. D. Wilkins, E. P. Steinberg, and R. R. Chasman, Phys. Rev. C 14, 1832 (1976).

[33] P. Paul and M. Thoennessen, Ann. Rev. Nucl. Part. Sci. 44, 65 (1994).

[34] G. Chaudhuri and S. Pal, Phys. Rev. C 63, 064603 (2001).

[35] P. Fröbrich, Prog. Theor. Phys. 154, 279 (2004). 